\title{
A longitudinal study of the lifestyle characteristics of adolescent with chronic fatigue symptoms
}

\author{
Takayoshi Yamada $^{1 *}$, Shinichi Demura ${ }^{2}$, Shunsuke Yamaji ${ }^{3}$, Shigeru Shimada ${ }^{4}$ \\ Faculty of Education and Regional Studies, University of Fukui, Fukui, Japan \\ Graduate School of Natural Science \& Technology, Kanazawa University, Ishikawa, Japan \\ Faculty of Medical Science, University of Fukui, Fukui, Japan \\ General Education Center (Natural Science \& Technology), Fukui National College of Technology, Fukui, Japan \\ *Corresponding author E-mail: yamadat@u-fukui.ac.jp
}

\begin{abstract}
Fatigue is a complicated, multifactorial disorder that is poorly understood. Till date, many studies conducted have crosssectionally analyzed the problem, but this fails to recognize the temporal nature of chronic fatigue that lasts over six months. This study aimed to examine the lifestyle characteristics of adolescents with chronic fatigue symptoms. In total, 462 healthy male students aged 15-19 years were enrolled to participate in a physical fitness test and surveys on subjective fatigue symptoms and lifestyle. They were assessed twice with a 1-year interval. Significant associations existed between both years in all parameters: for the low fatigue group $(\mathrm{V}=0.45-0.66)$, except for regularity of bedtime and seasoning and for the high fatigue group $(\mathrm{V}=0.45-0.73)$, except for regularity of the time of awakening, bedtime, and nutritional balance. Marked differences existed between the groups in the regularity of the time of awakening, nutritional balance, and seasoning (difference of $\mathrm{V} \geq 0.2$ ). All parameters tended to show inadequate lifestyle after a year in the high subjective fatigue symptom group. When compared with adolescents with low levels of fatigue, the lifestyles of adolescents with chronic fatigue changed more markedly and toward a more unhealthy or inadequate lifestyle.
\end{abstract}

Keywords: adolescent, chronic fatigue, lifestyle, longitudinal study, subjective fatigue

\section{Introduction}

Fatigue is defined as a specific state of pathological malaise that is induced by excessive glandular and psychological activity, with loss of physical function and the need for rest [1]. Chronic fatigue symptoms are diagnosed when the above stated symptoms persist for at least six months and when daily life is affected. Impairments of the nervous, endocrine, and immune systems are mainly listed as etiological factors in the development of chronic fatigue symptoms, and environmental stressors including allergy, infection, stress, or childhood abuse are possible critical factors [2]. However, no unified theory or pathology that can fully explain the symptom complex exists [3].

We hypothesize that chronic fatigue symptoms and similar symptoms occur because of chronic stress and a lack of healthy lifestyle. People with a lack of sleep, miss breakfast, and experience unpleasant awakening as well as those with a lower overall subjective sense of health tend to have higher subjective fatigue symptoms [4-6]. It is conceivable that people with chronic poor lifestyle and exercise habits may more likely complain of fatigue. However, it has been also reported that no clear relationship exists between lifestyle and exercise habits and symptoms of fatigue [7-10].

The differences between these findings could be because fatigue symptoms usually occur from chronic exposure to stress, with a consequent long-term disturbance in lifestyle. Chronic fatigue symptoms cannot be diagnosed if the above stated symptoms do not persist over a significant period. As a result, the cross sectional data obtained from many of the studies till date is unsuitable for assessing chronic fatigue symptoms. Collecting longitudinal data is much more suitable and essential in judging chronic fatigue. This study aimed to examine the lifestyle characteristics of adolescents with chronic fatigue symptoms based on longitudinal data. 


\section{Methods}

\subsection{Subjects}

In total, 462 healthy adolescent students, aged $15-19$ years $(16.0 \pm 0.9)$, were enrolled from the National College of Technology. Baseline height and weight were $170.5 \pm 5.7 \mathrm{~cm}$ and $60.1 \pm 9.5 \mathrm{~kg}$, respectively; physical characteristics were comparable with Japanese standard values. Informed consent was obtained from each subject following full explanation of the experiment. The experimental protocol was approved by the Ethics Committee on Human Experimentation of Faculty of Human Science, Kanazawa University (authorization number: 2012-14).

\subsection{Methods of assessment}

\subsubsection{Assessment of physical fitness and physique}

Physique was evaluated by height and weight. Physical fitness was measured on the basis of a fitness test from the Ministry of Education, Culture, Sports, Science and Technology in Japan. The following parameters were measured to assess physical fitness: grip strength, sit-ups, trunk anteflexion, repeated sideways jump, 20-meter shuttle run, 50-meter dash, standing long jump, and handball throw.

\subsubsection{Assessment of subjective symptoms of fatigue}

The subjective fatigue symptoms were assessed using the questionnaire proposed by Kobayashi et al. [11] to evaluate the fatigue of adolescent students. The questionnaire consists of the following six sub-scales (total 24 parameters): (1) difficulties with concentrated thinking; (2) languor; (3) loss of vigor; (4) loss of willingness; (5) drowsiness; and (6) uncomfortable physical feelings. Each fatigue sub-scale was then further categorized as follows [11-15]: (1) concentration, thinking faculties, and patience; (2) languor of the whole body or parts of it; (3) perturbation of conversation and loss of vigor; (4) irritation over moving and general distaste for activity; (5) drowsiness and hoping for attitude adjustment; and (6) eye strain and stiffness in shoulders, respectively. Subjects were asked to respond to each questionnaire parameter based on their feelings over the week prior to completion, using the following five-point rating scale [16]: no (1 point); not very ( 2 points); no preference ( 3 points); somewhat yes (4 points); yes (5 points). In addition, the scales were analyzed by assuming a five-point rating scale as an interval scale in this study.

\subsubsection{Assessment of lifestyle habits}

Lifestyle evaluation was based on the seven factors proposed by Belloc and Breslow [17]: (1) exercise habits; (2) eating habits; (3) sleeping; (4) maintaining adequate body-mass; (5) eating between meals; (6) drinking; and (7) smoking. Each lifestyle habit was then assessed on its own sub-scale as follows: exercise habits: exercise frequency [almost every day (more than 3 days/week], sometimes (1-2 days/week), occasionally (1-2 days/month), or never] and exercise time (less than $30 \mathrm{~min}, 30-60 \mathrm{~min}, 60-120 \mathrm{~min}$, or over $120 \mathrm{~min}$ ); eating habits: breakfast (eat every day, sometimes miss breakfast, or never eat breakfast), mealtimes (regularly eat three times a day, sometimes miss a meal, or irregular meals), food likes and dislikes (nothing, a little, or many), midnight snacks (often, sometimes, or never), amount of food consumed per meal (eats only a little, eats in moderation, or eats a lot), balanced nutrition (not generally, a little, or very), and seasoning (none, a little, or a lot); sleeping: duration of sleep (less than $6 \mathrm{~h}, 6-8 \mathrm{~h}$, or over $8 \mathrm{~h}$ ), regularity of the time of awakening (regular or irregular), and regularity of bedtime (regular or irregular); maintenance of proper weight: calculated body mass index (BMI) (less than 18.5, 18.5-25, or over 25) and percent body fat (less than $14 \%$, $14 \%-25 \%$, or over $25 \%$ ). Eating between meals (often, sometimes, or never) (Table 1). However, drinking and smoking were not included because the participants were not yet of a legal age.

\subsection{Measure and survey methods}

All tests were explained to subjects prior to assessment (the physical fitness test and questionnaires). The fitness tests were conducted in accordance with the standards laid down by the Ministry of Education, Culture, Sports, Science and Technology in Japan [18]. Fitness tests were conducted once a week in curricular classes delivered by experienced members of the health and physical education faculty between April and May in 2008 and 2009. The survey method described by Kobayashi et al. [11, 14] was used for the questionnaires. A subjective fatigue symptoms questionnaire was administered five times in each curricular class to allow for variation during the physical fitness test period. 


\subsection{Parameters}

Values recorded from the physical fitness tests were translated into point-based scores. The total scores of each subject were then calculated, and subjects were ranked from A to E based on their total points. The scores from the five subjective fatigue symptoms questionnaires were averaged and the total sub-scale scores were calculated.

\subsection{Determination of groups with high or low subjective fatigue symptoms}

The two survey years were grouped together, and the upper and lower quartiles of subjects reporting subjective fatigue were subsequently defined as high and low fatigue groups, respectively.

\subsection{Statistical analysis}

Cross tables of physical fitness decision and lifestyle parameters were described between the two study years for both the high and low fatigue groups. Cramer's V was calculated to examine the lifestyle in adolescents with chronic fatigue syndrome. Statistical significance was at a probability level of $<0.05$ and adjusted using the Bonferroni method.

\section{Results}

In total, 462 healthy adolescent students aged 15-19 years $(16.0 \pm 0.9)$ were enrolled from the National College of Technology. The baseline height and weight were $170.5 \pm 5.7 \mathrm{~cm}$ and $60.1 \pm 9.5 \mathrm{~kg}$, respectively, and their physical characteristics were comparable with Japanese standard values. Of these, 79 subjects each were analyzed in the high and low fatigue groups. The high subjective fatigue group had a mean age, height, and weight of $16.0 \pm 0.8$ years, 169.6 $\pm 5.9 \mathrm{~cm}$, and $59.1 \pm 9.6 \mathrm{~kg}$, respectively. The low subjective fatigue group had a mean age, height, and weight of 15.8 \pm 0.7 years, $171.3 \pm 5.8 \mathrm{~cm}, 59.0 \pm 9.2 \mathrm{~kg}$, respectively.

Table 1 shows two-way analysis of variance for physical characteristics and physical fitness scores between the low and high fatigue groups. The main changes over time occurred in height, weight, and percentage body fat, which were greater one year after baseline in both groups.

Table 1: Results of two-way analysis of variance for physical characteristics and physical fitness scores of both fatigue groups

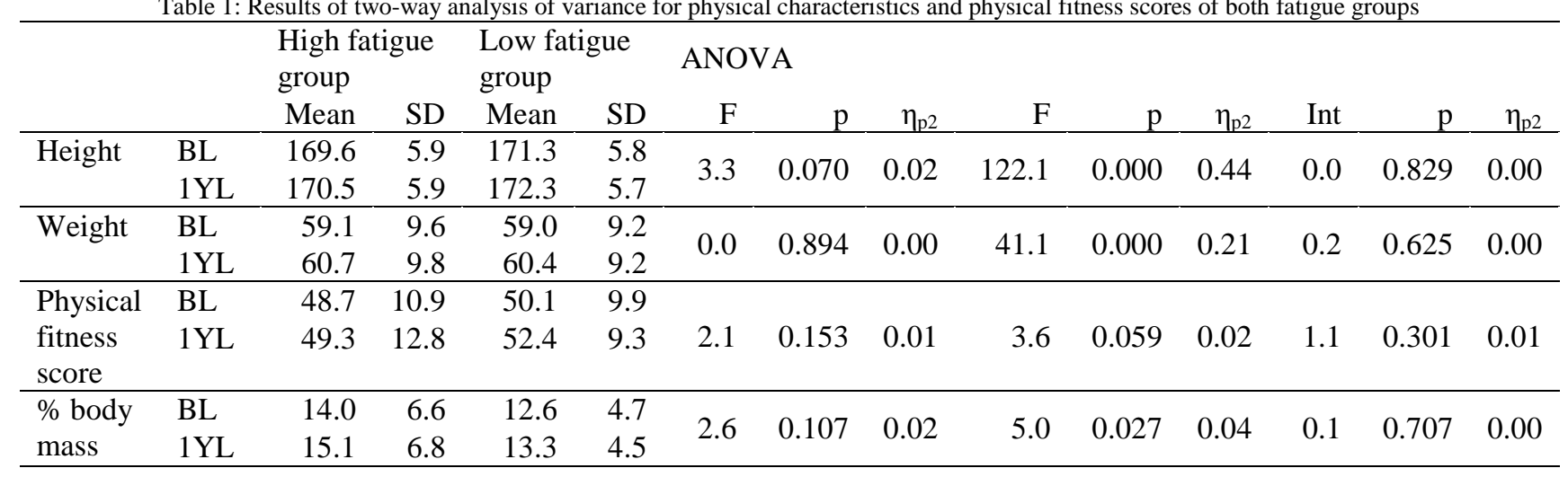

BL: Baseline, 1YL: 1 year later

Table 2 shows two-way analysis of variance for subjective fatigue symptoms score and sleep duration between the low and high fatigue groups. The high fatigue symptom group demonstrated a significantly higher subjective fatigue score after one year. In contrast, the low fatigue symptom group showed a small but significant decrease in fatigue. A significant effect was found with sleep duration, which was greater at baseline than at one year.

Table 3 shows the associations between responses at baseline and after one year for each lifestyle parameter between the low and high fatigue groups. Significant associations were found in all lifestyle parameters except for regularity of bedtime and seasoning in the low fatigue group $(\mathrm{V}=0.45-0.66)$. Significant associations were found in all lifestyle parameters except for regularity of the time of awakening, bedtime, and nutritional balance in the high fatigue group (V $=0.45-0.73)$. Breakfast, regularity of the time of awakening, eating between meals, nutritional balance, and seasoning were associated with large differences between the low and high fatigue groups, and the $\mathrm{V}$ values were higher in the low fatigue group than in the high fatigue group. 
Table 2: Results of two-way analysis of variance for subjective fatigue symptoms score and sleeping time of both fatigue groups

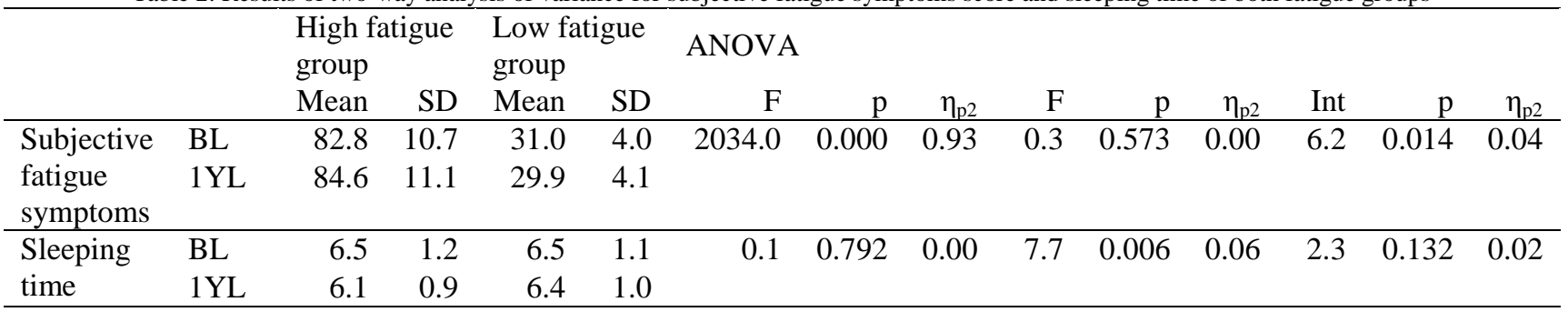

BL: Baseline, 1YL: 1 year later

Table 3: Correlations between responses at baseline and after 1 year of each lifestyle item in both groups

\begin{tabular}{lrrrrrrrrrr}
\hline & \multicolumn{4}{c}{ Low fatigue group } & \multicolumn{4}{c}{ High fatigue group } \\
& $\chi^{2}$ & $\mathrm{df}$ & $\mathrm{p}$ & $\mathrm{V}$ & $\mathrm{p}$ & $\chi^{2}$ & $\mathrm{df}$ & $\mathrm{p}$ & $\mathrm{V}$ & $\mathrm{p}$ \\
\hline Exercise frequency & 66.1 & 9 & 0.000 & 0.53 & $0.000^{*}$ & 64.4 & 9 & 0.000 & 0.52 & $0.000^{*}$ \\
Exercise time & 59.4 & 12 & 0.000 & 0.50 & $0.000^{*}$ & 62.1 & 12 & 0.000 & 0.51 & $0.000^{*}$ \\
Breakfast & 29.8 & 2 & 0.000 & 0.61 & $0.000^{*}$ & 33.6 & 6 & 0.000 & 0.46 & $0.000^{*}$ \\
Physical fitness & 108.9 & 16 & 0.000 & 0.59 & $0.000^{*}$ & 98.5 & 16 & 0.000 & 0.56 & $0.000^{*}$ \\
Time of awakening & 15.2 & 2 & 0.000 & 0.48 & $0.000^{*}$ & 2.0 & 2 & 0.362 & 0.17 & $0.362 \mathrm{~ns}$ \\
Bedtime & 3.3 & 2 & 0.190 & 0.23 & $0.190 \mathrm{~ns}$ & 4.3 & 1 & 0.039 & 0.25 & $0.039 \mathrm{~ns}$ \\
Eating habit & 12.9 & 2 & 0.002 & 0.45 & $0.002^{*}$ & 40.1 & 4 & 0.000 & 0.54 & $0.000^{*}$ \\
Like/dislike & 56.6 & 4 & 0.000 & 0.66 & $0.000^{*}$ & 74.1 & 4 & 0.000 & 0.73 & $0.000^{*}$ \\
Eating between meals & 45.2 & 4 & 0.000 & 0.59 & $0.000^{*}$ & 28.8 & 4 & 0.000 & 0.45 & $0.000^{*}$ \\
Midnight snack & 30.4 & 4 & 0.000 & 0.48 & $0.000^{*}$ & 40.1 & 4 & 0.000 & 0.53 & $0.000^{*}$ \\
Amount of food consumed & 20.4 & 2 & 0.000 & 0.56 & $0.000^{*}$ & 31.9 & 4 & 0.000 & 0.48 & $0.000^{*}$ \\
Nutrient balance & 37.5 & 4 & 0.000 & 0.54 & $0.000^{*}$ & 11.2 & 4 & 0.025 & 0.28 & $0.025 \mathrm{~ns}$ \\
Seasoning & 8.5 & 4 & 0.075 & 0.26 & $0.075 \mathrm{~ns}$ & 52.0 & 4 & 0.000 & 0.61 & $0.000^{*}$ \\
\hline
\end{tabular}

Tables 4-7 display cross tables of the parameters and indicate marked differences in associations between responses at baseline and after one year for each lifestyle parameter. Lifestyle variation in the low fatigue group was smaller than that in the high fatigue group for all parameters. Moreover, more students transferred from an adequate lifestyle to an inadequate lifestyle in the high fatigue group. This trend was also similar with regard to the other parameters.

Table 4 Cross table of the items regarding "Breakfast" between responses at baseline and after one year

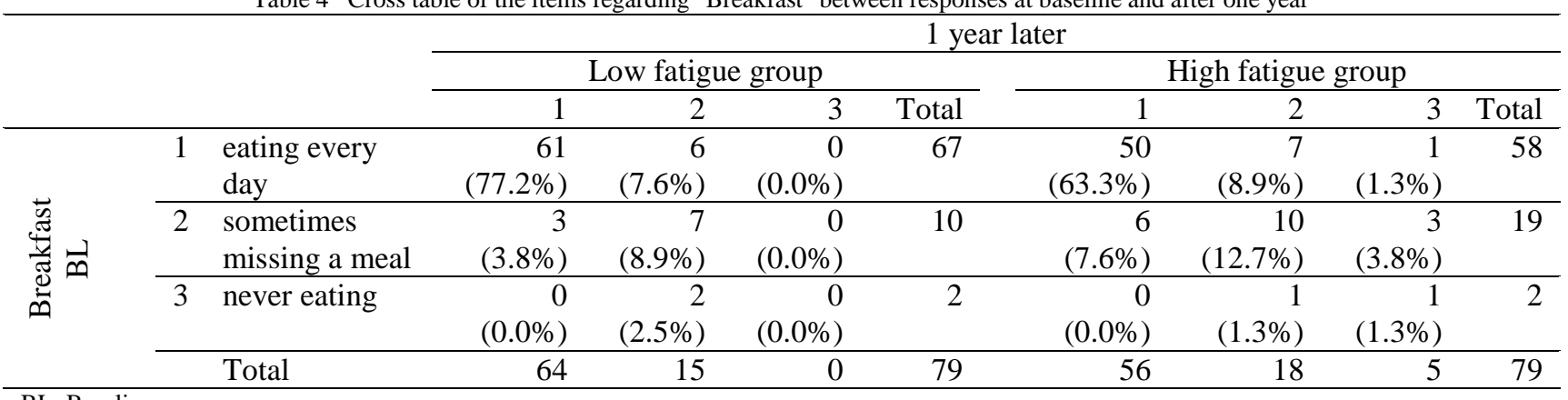

Table 5 Cross table of the items regarding "Regularity of getup time" between responses at baseline and after one year

\begin{tabular}{|c|c|c|c|c|c|c|c|c|}
\hline & & & \multicolumn{6}{|c|}{1 year later } \\
\hline & & & \multicolumn{3}{|c|}{ Low fatigue group } & \multicolumn{3}{|c|}{ High fatigue group } \\
\hline & & & 1 & 2 & Total & 1 & 2 & Total \\
\hline \multirow{3}{*}{ 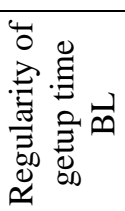 } & 1 & regular & $\begin{array}{r}61 \\
(93.8 \%)\end{array}$ & $\begin{array}{r}1 \\
(1.5 \%)\end{array}$ & 62 & $\begin{array}{r}60 \\
(92.3 \%)\end{array}$ & $\begin{array}{r}5 \\
(7.7 \%)\end{array}$ & 65 \\
\hline & 2 & irregular & $\begin{array}{r}2 \\
(3.1 \%)\end{array}$ & $\begin{array}{r}1 \\
(1.5 \%)\end{array}$ & 3 & $\begin{array}{r}3 \\
(4.6 \%)\end{array}$ & $\begin{array}{r}1 \\
(1.5 \%)\end{array}$ & 4 \\
\hline & & Total & 63 & 2 & 65 & 63 & 6 & 69 \\
\hline
\end{tabular}

BL: Baseline 
Table 6: Cross table of the items regarding "Eating between meals" between responses at baseline and after one year

\begin{tabular}{|c|c|c|c|c|c|c|c|c|c|c|}
\hline & & & \multicolumn{8}{|c|}{1 year later } \\
\hline & & & \multicolumn{4}{|c|}{ Low fatigue group } & \multicolumn{4}{|c|}{ High fatigue group } \\
\hline & & & 1 & 2 & 3 & Total & 1 & 2 & 3 & Total \\
\hline \multirow{5}{*}{ 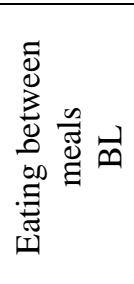 } & 1 & often & 12 & 3 & 2 & 17 & 16 & 8 & 0 & 24 \\
\hline & & & $(18.5 \%)$ & $(4.6 \%)$ & $(3.1 \%)$ & & $(22.9 \%)$ & $(11.4 \%)$ & $(0.0 \%)$ & \\
\hline & 2 & sometimes & $\begin{array}{r}7 \\
(10.8 \%)\end{array}$ & $\begin{array}{r}28 \\
(43.1 \%)\end{array}$ & $\begin{array}{r}3 \\
(4.6 \%)\end{array}$ & 38 & $\begin{array}{r}5 \\
(7.1 \%)\end{array}$ & $\begin{array}{r}31 \\
(44.3 \%)\end{array}$ & $\begin{array}{r}2 \\
(2.9 \%)\end{array}$ & 38 \\
\hline & 3 & never & $\begin{array}{r}0 \\
(0.0 \%)\end{array}$ & $\begin{array}{r}2 \\
(3.1 \%)\end{array}$ & $\begin{array}{r}8 \\
(12.3 \%)\end{array}$ & 10 & $\begin{array}{r}0 \\
(0.0 \%)\end{array}$ & $\begin{array}{r}6 \\
(8.6 \%)\end{array}$ & $\begin{array}{r}2 \\
(2.9 \%)\end{array}$ & 8 \\
\hline & & Total & 19 & 33 & 13 & 65 & 21 & 45 & 4 & 70 \\
\hline
\end{tabular}

BL: Baseline

Table 7 Cross table of the items regarding "Nutrient balance" between responses at baseline and after one year

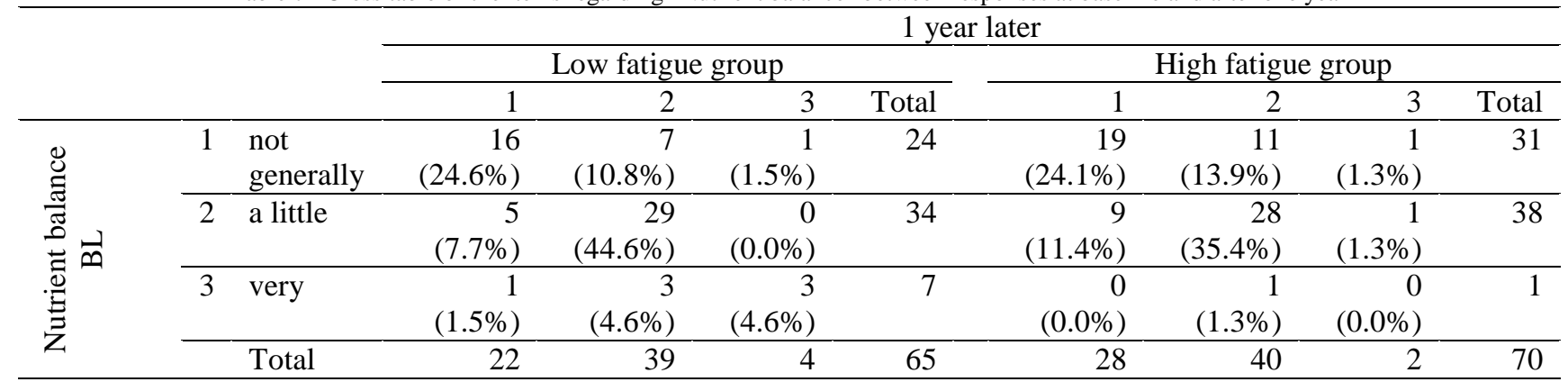

\section{Discussion}

Subjective fatigue symptom scores were significantly higher at both baseline and one year later in the low fatigue group than in the high fatigue group. Consistent with the definition of fatigue by Kidani and Watanabe (2001), the high fatigue group in this study suffered from relatively high levels of fatigue symptoms for a year from baseline. However, they attended school and curricular class. In spite of encountering difficulties in activities of daily living, no intervention was necessary. In 2010 (van't Leven et al.), 22,500 people were surveyed to clarify the frequency of fatigue symptoms in the general population; the survey obtained 9,062 valid responses (43\%), reporting that people were divided into groups with no symptoms $(64.4 \%)$, short-term fatigue (4.9\%), chronic fatigue (30.5\%), chronic fatigue-like symptoms (1.0\%), and many requiring rest because of fatigue. The diagnostic criteria differed between the present study and the van't Leven study; we defined the high fatigue groups as the upper quartile of subjects complaining of high levels of subjective fatigue. This is comparable with the $30.5 \%$ patients with chronic fatigue, the $1 \%$ with chronic fatigue-like complaints, and the general population requiring rest because of fatigue. However, the present high fatigue group was judged to have higher levels of fatigue symptoms. Therefore, the cohort in this study is probably adequate to clarify the lifestyle characteristics related to chronic fatigue.

The associations between responses at baseline and one year in the lifestyle categories were higher in the low fatigue group than in the high fatigue group. These tendencies were most marked in breakfast, regularity of the time of awakening, eating between meals, and nutritional balance, suggesting that lifestyle in the low fatigue group remains largely unchanged after one year. Moreover, the low fatigue group maintained a healthier lifestyle in all lifestyle parameters when compared with the high fatigue group (Tables 4-7), and these tendencies were similar across all parameters. Unger et al. (2004) surveyed 399 patients with chronic fatigue symptoms about fatigue, health, and sleep to clarify their sleeping characteristics and reported that $81.4 \%$ of these patients suffered from some degree of sleep disturbance, and as a result they had subjectively significantly decreased health . Although sleep duration, regularity of the time of awakening, and bedtime were all assessed in this study, a difference was only found between the groups for the regularity of the time of awakening. A marked difference may not be found because the fatigue levels in our subjects were relatively lower than those in the study by Unger et al. (2004); however, it is suggested that adequate sleep positively affects fatigue. For example, Belloc and Breslow (1972) advocate that it is desirable to sleep for 7-8 h per day. The quality and quantity of sleep was not considered to be adequate in this study, and no difference was found 
between the groups. Based on this analysis, although sleep duration may also be important, maintaining a consistent time of awakening may be a more important factor in controlling symptoms of fatigue amongst adolescents.

Tendencies as those similar to sleeping were also found with regard to breakfast, eating between meals, and nutritional balance. To clarify the lifestyle characteristics associated with fatigue, Goedendorp et al. (2009) surveyed lifestyle (smoking, drinking, ingestion of fat, dietary fiber, fruit, and vegetables), BMI, and fatigue symptoms among 247 patients with chronic fatigue symptoms. They reported that $23 \%$ smoked and $32 \%$ had an unhealthy BMI, but none had an unhealthy alcohol intake. A majority were found to have an unhealthy food intake: $70 \%$ had unhealthy fat, fruit, and vegetable intakes, while $95 \%$ had unhealthy levels of fiber intake. Compared with the general population, significantly fewer fatigue patients were overweight. However, no relationship was found between an unhealthy lifestyle and the degree of fatigue, and it was concluded that the lifestyle of patients with chronic fatigue was healthier than that of the general population. Compared with a non-fatigued group, the group complaining of fatigue had the following characteristics: more females [odds ratio (OR), 1.9], more obese (OR, 4.1), higher use of analgesic (OR, 7.8), higher alcohol intake (OR, 0.4), healthier food intake (OR, 0.5), and fewer were physically less active (OR, 0.1). Mirically (1997) conducted a follow-up survey in 28 patients to evaluate the outcomes of chronic fatigue symptoms and to determine the factors associated with a better prognosis but failed to report any major prognostic factors, although they did find an association with marital status. To date, no consistent lifestyle factors have been identified as causative in the development of fatigue symptoms. However, in this study, some lifestyle differences were identified between the high and low fatigue groups. The findings obtained in this study, through a longitudinal rather than a cross-sectional design, may assist with informed decision making and the reduction of symptoms of fatigue.

\section{Conclusion}

When compared with adolescents with low levels of fatigue, the lifestyles of adolescents with chronic fatigue symptoms changed more markedly, and toward a more unhealthy or inadequate lifestyle, particularly in terms of breakfast consumption, consistency of the time of awakening, eating between meals and nutritional balance.

\section{Acknowledgements}

The authors have no financial disclosures to make on this paper and contributed to all aspects of this paper and there is no sponsor to this study.

\section{References}

[1] M. Inoue, H. Kuratsune, Y. Watanabe, Science of fatigue -Warning on the unsleeping modern society-, Kodansha Scientific, Tokyo, 2001.

[2] R.R. Dietert, J.M. Dietert, Possible role for early-life immune insult including developmental immunotoxicity in chronic fatigue syndrome (CFS) or myalgic encephalomyelitis (ME), Toxicology 247 (1) (2008) 61-72.

[3] V.C. Lombardi, F.W. Ruscetti, J. Das Gupta J, et al., Detection of an Infectious Retrovirus, XMRV, in Blood Cells of Patients with Chronic Fatigue Syndrome, Science 326 (5952) (2009) 585-589.

[4] S. Kadota, Relationship between subjective symptoms of fatigue and living behavior in senior high school students--a study by quantification method II, Japanese Journal of School Health 32 (1990) 239-247.

[5] S. Kadota, Examination of students' health care administration -living conditions and subjective fatigue symptoms-, Japanese journal of school health 20 (1978) 286-291.

[6] M. Takakura, Relationship between type a behavior pattern and fatigue symptoms, life style in university students, Japanese Journal of School Health 35 (1993) 484-491.

[7] S. Demura, T. Yamada, S. Shimada et al., Influence of exercise habits and physical fitness level on subjective fatigue symptoms in adolescent students, Health 3 (2011) 20-25.

[8] T. Yamada, S. Demura S, Influence of exercise habits and physical fitness level on subjective fatigue symptoms in adolescents, Human Performance Measurement 9 (2012) 1-8.

[9] M. van't Leven, G.A. Zielhuis, J.W. van der Meer et al., Fatigue and chronic fatigue syndrome-like complaints in the general population, European Journal of Public Health 20 (2010) 251-257.

[10] M.M. Goedendorp, H. Knoop, G.M. Schippers et al., The lifestyle of patients with chronic fatigue syndrome and the effect on fatigue and functional impairments, Journal of Human Nutrition and Dietetics 22 (2009) 226-231.

[11] H. Kobayashi, S. Demura, F. Goshi et al., Construction of a subjective fatigue scale for adolescent students, Japanese Journal of Public Health 47 (2000) 638-645.

[12] S. Demura, H. Kobayashi, J. Matsuzawa, Examination of proposed items for a fatigue questionnaire for students based on subjective symptoms, Japanese Journal of Public Health 44 (1997) 427-439.

[13] H. Kobayashi, S. Demura, F. Goshi, Sex differences of fatigue in young men and women-From the view point of experience of awareness and cognitive appraisal-, Japanese Journal of Physical Fitness and Sports Medicine 47 (1998) 581-592. 
[14] S. Demura, H. Kobayashi, S. Sato, Examination of validity of the subjective fatigue scale for young adults, Japanese Journal of Public Health 48 (2000) 76-84.

[15] H. Kobayashi, S. Demura, S. Sato, Relationship between a subjective fatigue scale for young adults and the subjective symptoms index, Japan Journal of physical Education, Health and Sport Sciences 46 (2001) 35-46.

[16] H. Kobayashi, S. Demura, Gender differences in the degree of complaints and frequency of subjective symptoms of fatigue and relationships among their domains in high school students, School Health 2 (2006) 33-37.

[17] N.B. Belloc, L. Breslow, Relationship of physical health status and health practices. Preventive Medicine 1 (3) (1972) 409-421.

[18] Ministry of Education, Culture, Sports, Science and Technology in Japan, Deployment plan of new physical fitness test (subject of 20-64yr), http://www.mext.go.jp/component/a_menu/sports/detail/__icsFiles/afieldfile/2010/07/30/1295079_03.pdf 